\title{
The econophysical turn and economic complexity
}

\author{
Christophe Schinckus ${ }^{1,2 *}$ \\ 1 School of Management, University of Leicester, Leicester, UK \\ 2 Department of History and of Philosophy of Science, University of Cambridge, Cambridge, UK \\ *Correspondence: cs354@le.ac.uk \\ Edited by: \\ Wei-Xing Zhou, East China University of Science and Technology, China \\ Reviewed by: \\ Boris Podobnik, Faculty of Civil Engineering, Croatia \\ Benjamin Miranda Tabak, Universidade Catolica de Brasilia, Brazil \\ Sai-Ping Li, Academia Ainica, Taiwan
}

Keywords: econophysics, agent based modeling, complexity, economics, interdisciplinarity

\section{A commentary on}

What has econophysics ever done for us? by Buchanan M. (2013). Nat. Phys. 9, 317. doi: $10.1038 /$ nphys2648

This general commentary extends the excellent paper written by Buchanan in a previous issue of Nature Physics ("What has econophysics ever done for us?" Nature Physics, 9, 317, 2013) in which the author proposed a list of "good things" related to contribution of econophysics. Rather than detailing the major results of econophysics which could be potentially interesting for economics (see point 1-7 in [1]), this note extends the 8 th point proposed by Buchanan by emphasizing the methodological contribution of the first to the latter for a better understanding of economic complexity.

Econophysics is a new interdisciplinary field that emerged in the shadow of physics. Roughly speaking, econophysics refers to the application of physical tools $\backslash$ models for describing the evolution of complex economic systems such as financial markets, inter-firm transactions or credit networks. The increasing number of papers in physics journal dedicated to econophysics contributed to the official recognition of the field by the Physics and Astrophysics Classification Scheme (PACS) since it is nowadays an official subcategory of physics under the code $89.65 \mathrm{Gh}$. Like the vast majority of emerging interdisciplinary fields, econophysics generated a lot of debates leading some authors [1] to question the contribution of econophysics or even to deny its potential contribution [2].
Although the economic mainstream usually modeled the market behavior as an addition of agents' behaviors, econophysicists consider that social systems are "more than the sum of their parts" by emphasizing the fact that economics is concerned with emergent phenomena and complex systems. Conceptually, this idea is nothing new since Hayek [3] dealt with this kind of emergent phenomena in society. Hayek defined self-regulating emergence as a phenomenon based on multiple agents interacting in such a way as to generate the macro-properties of economic systems. Hayek called "Catallaxy" this emergence of a self-order in the economic systems that he presented more as a political argument than a scientific one since he did not give an operational definition to this phenomenon. In contrast to Hayek, econophysicists develop an operational form characterizing the emergence in economic complex systems without giving a political argument.

By using a formal Chomskian definition of language (as a complex adaptative system), Loreto and Steels [4] made statistical physics relevant for studying its emergence and evolution. The emergence of money is another topic dealt by physicists: Bak et al. [5] showed that emergence of money is a history (time) dependent process while Donangelo and Sneppen [6] showed how the emergence of money can be described through a scaling relation between the number of exchanges. In the same vein, Feng et al. [7] initially identified macro-patterns governing the evolution of financial markets in order to define the calibration of the rules governing interactions between agents. This combination between a macro (top-down) and a micro (bottom up) analysis of complex economic systems is the major difference with agentbased modeling in economics where only a micro (bottom-up) is implemented.

Works mentioned here (among others) are evidence that econophysics is more than an importation of concepts by unemployed physicists as one can find on social networks ${ }^{1}$. By combining a phenomenological (macro) analysis of complex economic systems with an agent-based approach (micro), econophysicists provide an algorithmically derivable emergence clarifying the gap between the macro and the micro scales. Because this way of dealing with economic complexity provides micro-foundations (i.e., potentially behavioral explanations) to the statistical regularities that emerge at the macro-level of socio-economic systems, it can significantly contribute to a better understanding of old issues studies in economics such as emergent properties. Moreover, the framework proposed by econophysicists is conceptually in line with the classical notion of reduction used in economics (and in physics [8]) and the idea of a derivable emergence is compatible with the liberal and individualist view of systems enhanced by economists since the large numbers of interactions between actors are assumed to result from "free will" (this link between probabilistic nature of the laws of the statistical (and quantum) mechanical and free will has been summarized by Bouchaud and Cont [9]).

${ }^{1}$ http://blog.physicsworld.com/2008/12/18/by-joao-m edeiros-ive-just/ or http://www.econjobrumors.com/ topic/econophysics-2 
In conclusion, I agree with Buchanan when he wrote that econophysics did not degenerate into irrelevance and I would go beyond by arguing that the econophysical turn contributes to the "scientific revolution" demanded by some authors $[10,11]$ in which they invite economists to integrate conceptual tools coming from statistical physics in their agent-based modeling. This commentary is an enabler for an increasing collaboration between economics and econophysics which, though coming from two different scientific cultures, have more in common than one could expect [12].

\section{REFERENCES}

1. Buchanan M. What has econophysics ever done for us? Nat Phys. (2013) 9:317. doi: $10.1038 /$ nphys 2648

2. Durlauf S. Complexity and empirical economics. Econ J. (2005) 115:225-43. doi: 10.1111/j.14680297.2005.01003.x

3. Hayek F. Scientism and the study of society. Economica (1942) 9:267-91.
4. Loreto V, Steels L. Social dynamics: Emergence of language. Nature Physics (2007) 3:758-60. doi: 10.1038/nphys770

5. Bak P, Paczuski M, Shubik M. Price Variations in a Stock Market with many agents. Physica A (1997) 246:430-53. doi: 10.1016/S0378-4371(97) 00401-9

6. Donangelo R, Sneppen K. Self-organization of value and demand. Physica A (2000) 276:572580. doi: 10.1016/S0378-4371(99)00473-2

7. Feng L, Li B, Podobnik B, Preis T, Stanley E. Linking agent-based models and stochastic models of financial markets. Proc Natl Acad Sci USA (2012) 109:8388-93. doi: 10.1073/pnas. 1205013109

8. Schinckus C. Stylized Facts and Econophysics. Working paper, University of Leicester, Leicester (2014).

9. Bouchaud JP, Cont R. A Langevin approach to stock market fluctuations and crashes. Eur Phys J B (1998) 6:543-50. doi: 10.1007/s1005 10050582

10. Bouchaud JP. Economics needs a scientific revolution. Nature (2009) 455:1181. doi: 10.1038/4551181a

11. Farmer D, Foley D. The Economy needs agentbased modelling. Nature (2009) 460:685-6. doi: $10.1038 / 460685 \mathrm{a}$
12. Jovanovic F, Schinckus C. Towards a transdisciplinary econophysics. J Econ Methodol. (2013) 20:164-83. doi: 10.1080/1350178X.2013. 801561

Conflict of Interest Statement: The author declares that the research was conducted in the absence of any commercial or financial relationships that could be construed as a potential conflict of interest.

Received: 14 May 2014; paper pending published: 09 June 2014; accepted: 18 June 2014; published online: 07 July 2014.

Citation: Schinckus C (2014) The econophysical turn and economic complexity. Front. Phys. 2:41. doi: 10.3389/fphy.2014.00041

This article was submitted to Interdisciplinary Physics, a section of the journal Frontiers in Physics.

Copyright (C) 2014 Schinckus. This is an open-access article distributed under the terms of the Creative Commons Attribution License (CC BY). The use, distribution or reproduction in other forums is permitted, provided the original author(s) or licensor are credited and that the original publication in this journal is cited, in accordance with accepted academic practice. No use, distribution or reproduction is permitted which does not comply with these terms. 